Pacific Journal of Mathematics

SOME RESULTS ON LACUNARY WALSH SERIES 


\title{
SOME RESULTS ON LACUNARY WALSH SERIES
}

\author{
JoHN COURY
}

\begin{abstract}
It is known that if a lacunary trigonometric series converges to 0 on a set of positive measure, then the series vanishes identically. In the present paper, the following analogue for the Walsh system is proved: a lacunary Walsh series which converges to 0 almost everywhere is identically zero.
\end{abstract}

In particular, let $S(x)=\sum_{1}^{\infty} c_{k} w_{n_{k}}(x)$ be a lacunary Walsh series, with $n_{k+1} / n_{k} \geqq q>1$ for each $k$. We prove that if $S(x)$ converges to 0 on a set of positive measure, or on a set of the second category having the property of Baire, then the series is a finite sum. If $S(x)$ converges to 0 on a set of sufficiently large measure (the measure depending only on the degree of lacunarity $q$ ), then $S(x)$ is identically zero. Hence we prove that the only lacunary Walsh series converging to 0 almost everywhere is the identically zero series. Finally, sufficient conditions are given for a set to be a set of uniqueness for lacunary Walsh series.

1. Preliminaries. If $x \in[0,1[$ has the dyadic development $\sum_{k=1}^{\infty} x_{k} 2^{-k}$, where $x_{k}$ is 0 or 1 , then the $(k-1)$ st Rademacher function $r_{k-1}$ evaluated at $x$ has the value $(-1)^{x_{k}}$. (For dyadic rationals in $[0,1$, which have two such expansions, we agree to take the finite development.) If we write a positive integer $n$ as $2^{n_{1}}+2^{n_{2}}+\cdots+2^{n_{v}}$, where $n_{1}>n_{2}>\cdots>n_{v} \geqq 0$, then the $n$th Walsh function is given by $w_{n}=r_{n_{1}} \cdot r_{n_{2}} \cdot \cdots \cdot r_{n_{v}}$ (following Paley's modification). Define $w_{0}(x) \equiv 1$; then the functions $\left\{w_{n}\right\}_{n=0}^{\infty}$ form a complete orthonormal set on $[0,1[$. For $n \geqq 1, w_{n}$ is plainly discontinuous.

Fine [2] has shown that the Walsh functions may be identified with the full character group $\left\{w_{n}^{*}\right\}$ of $2^{\omega}$, where $2^{\omega}$ denotes the countable product of the two-element group $\{0,1\}$. For $\boldsymbol{x}=\left(x_{1}, x_{2}, \cdots\right)$ in $2^{\omega}$, define $w_{n}^{*}(\boldsymbol{x})=r_{n_{1}}^{*}(\boldsymbol{x}) \cdot \cdots \cdot r_{n_{v}}^{*}(\boldsymbol{x})$, where $n=2^{n_{1}}+\cdots 2^{n_{v}}$ and $r_{k-1}^{*}(\boldsymbol{x})=(-1)^{x_{k}}$. To simplify notation, we shall suppose henceforth that $r_{k-1}^{*}(\boldsymbol{x})=(-1)^{x_{k-1}}$, where $k \geqq 2$.

I.et $\phi(x)=\sum_{k=1}^{\infty} x_{k} 2^{-k}$, where $\boldsymbol{x}=\left(x_{1}, x_{2}, \cdots\right) \in 2^{\omega}$. Then $\phi(x)$ is a continuous measure-preserving map of $2^{\omega}$ onto [0,1[ but is not injective: a dyadic rational in [0,1[ is the image of two points in $2^{\omega}$. If $\mathscr{F}$ denotes the set of sequences in $2^{\omega}$ that are eventually 1 , then 
$\phi(\boldsymbol{x})$ is one-to-one on the complement of $\mathscr{F}$ and $\phi(\boldsymbol{x})$ maps $\mathscr{F}$ onto the dyadic rationals of $[0,1[$. This 'exceptional' set $\mathscr{F}$ corresponds to the infinite expansion of dyadic rationals; $\mathscr{F}$ is plainly countable and hence has Haar measure 0.

With a Walsh series $\sum c_{n} w_{n}(x)$ on [0,1[, we may associate the corresponding 'Walsh' series $\sum c_{n} w_{n}^{*}(\boldsymbol{x})$ on $2^{\omega}$, where $\boldsymbol{x}$ is such that $\phi(\boldsymbol{x})=x$. Clearly, if we neglect the points of $\mathscr{F}$, the former series converges to a value $c$ when and only when the latter series also converges to $c$.

Thus, since $\mathscr{F}$ is of the first category and has Haar measure zero in $2^{\omega}$, it suffices to prove the theorems stated in the introduction on the group $2^{\omega}$, using lacunary series of the form $S^{*}(\boldsymbol{x})=\sum c_{k} w_{n_{k}}^{*}(\boldsymbol{x})$. The advantage is that the functions $w_{n}^{*}$ are continuous with respect to the usual (product) topology of $2^{\omega}$; however, all of the results of $\S \S 2$ and 3 are valid for lacunary Walsh series defined on the interval [0,1[. Henceforth we write $S(\boldsymbol{x})$ for $S^{*}(\boldsymbol{x})$ and $w_{n}$ for $w_{n}^{*}$.

2. The main results. A Walsh series $S(\boldsymbol{x})=\sum_{k=1}^{\infty} c_{k} w_{n_{k}}(\boldsymbol{x})$ is called $q$-lacunary if $n_{k+1} / n_{k} \geqq q>1$ for all $k$, where $q$ is the supremum of all such numbers.

Lemma. Let $S(\boldsymbol{x})$ be a q-lacunary Walsh series, with $q \geqq 2$. Suppose that $S(\boldsymbol{x})$ converges to 0 (or is constant) on a set $E$ of Haar measure exceeding 1/2. Then $S(\boldsymbol{x})$ vanishes identically.

Proof. Since $q \geqq 2$, each $n_{k}$ contains a power of 2 greater than each power of 2 in $n_{k-1}$. Thus the series $S(x)$ is simply a series $\sum c_{k} X_{k}$, where the $X_{k}$ are independent random variables of the Bernoulli type. This latter series may in turn be viewed as a Rademacher series $R(\boldsymbol{x})=\sum_{k=1}^{\infty} c_{k} r_{k}(\boldsymbol{x})$. Thus, as far as measure is concerned, the series $R(x)$ and $S(x)$ have the same properties, and so it suffices to prove the lemma for Rademacher series.

For an arbitrary positive integer $N$, let $\boldsymbol{u}_{N}$ denote the point in $2^{\omega}$ with entry 1 in the Nth coordinate and 0's elsewhere. Since adding $\boldsymbol{u}_{N}$ to a point $\boldsymbol{x} \in 2^{\omega}$ affects only the $N$ th coordinate of $\boldsymbol{x}$, it follows that $r_{k}\left(\boldsymbol{x}+\boldsymbol{u}_{N}\right)=r_{k}(\boldsymbol{x})$ for every $k \neq N$, and $r_{N}\left(\boldsymbol{x}+\boldsymbol{u}_{N}\right)=-r_{N}(\boldsymbol{x})$.

Let $\mu$ denote (normalized) Haar measure on $2^{\omega}$. Since $\mu(E)>1 / 2$, the set $E \cap\left(E+\boldsymbol{u}_{N}\right)$ has positive measure for every $N$, where $E+\boldsymbol{u}_{N}=\left\{\boldsymbol{x}+\boldsymbol{u}_{N}: \boldsymbol{x} \in E\right\}$. Thus there exists $z \in E$ such that $z+\boldsymbol{u}_{N} \in E$. If $R_{n}$ denotes the $n$th partial sum of $R$, we have

and

$$
\begin{array}{ll}
R_{n}\left(z+\boldsymbol{u}_{N}\right)-R_{n}(z)=0 & \text { for } n<N, \\
R_{n}\left(z+\boldsymbol{u}_{N}\right)-R_{n}(z)=-2 c_{N} r_{N}(z) & \text { for } n \geqq N .
\end{array}
$$

Passing to the limit as $n \rightarrow \infty$, we obtain $-2 c_{N} r_{N}(z)=0$, whence 
$c_{N}=0$. Since $N$ was arbitrary, we conclude that $R(\boldsymbol{x})$ vanishes identically.

It is well-known (see, for example, [1, vol. II, p. 233]) that for a given $q>1$, any lacunary sequence of integers can be written as the finite union of pairwise disjoint lacunary subsequences each of whose degree of lacunarity is at least $q$. The least number of $q$-lacunary subseries, with $q \geqq 2$, into which a given lacunary series can be partitioned will be called the index of 2-lacunarity of the series. We now generalize the result of the lemma to arbitrary lacunary Walsh series.

Theorem 1. Let $S(\boldsymbol{x})$ be a lacunary Walsh series, and let $M$ denote its index of 2-lacunarity. Suppose that $S(\boldsymbol{x})$ converges to 0 (or is constant) on a set $E$ with $\mu(E)>1-1 / 2^{H}$. Then $S(\boldsymbol{x})$ is identically zero.

Proof. By induction on $M$. For $M=1$, the result follows from the lemma. Thus assume the theorem is true for an index $M-1$; write $S(\boldsymbol{x})=\sum_{i=1}^{M} S_{i}(\boldsymbol{x})$, where $S_{i}(\boldsymbol{x})=\sum_{k=1}^{\infty} c_{i, k} w_{n_{i, k}}(\boldsymbol{x})$ and for each fixed $i$ the sequence $\left(n_{i, k}\right\}$ is $q$-lacunary with $q \geqq 2$.

For a positive integer $N$, write $N=2^{N_{1}}+\cdots+2^{N_{v}}$, with $N_{1}>N_{2}>\cdots>N_{v} \geqq 0$. Then for $\boldsymbol{x} \in 2^{\omega}$, we shall say that the coordinates of $\boldsymbol{x}$ which "correspond to $N$ " are the $N_{i}$ th entries, $i=1,2, \cdots, v$.

We may suppose that $n_{M, 1}<n_{1,1}$. Since $n_{1,1}$ has a power of 2 that $n_{M, 1}$ does not, choose $z_{1}$ for $n_{1,1}$ so that in those coordinates of $z_{1}$ corresponding to $n_{M, 1}$ there are an odd number of 1 's, and in the coordinates corresponding to every $n_{1, k}, k \geqq 1$, there are an even number of 1's. For arbitrary $\boldsymbol{x} \in 2^{\omega}$, we have

$$
\begin{aligned}
S\left(\boldsymbol{x}+z_{1}\right)-S(\boldsymbol{x})= & \sum_{k=1}^{\infty} c_{1, k}\left[w_{n_{1, k}}\left(z_{1}\right)-1\right] w_{n_{1, k}}(\boldsymbol{x}) \\
& +\sum_{i=2}^{M} \sum_{k=1}^{\infty} c_{i, k}\left[w_{n_{i, k}}(\boldsymbol{x}+\boldsymbol{z})-w_{n_{i, k}}(\boldsymbol{x})\right] \\
= & \sum_{i=2}^{M} \sum_{k=1}^{\infty} c_{i, k}\left[w_{n_{i, k}}\left(\boldsymbol{z}_{1}\right)-1\right] w_{n_{i, k}}(\boldsymbol{x}),
\end{aligned}
$$

since $w_{n_{1, k}}\left(z_{1}\right)=1$ for every $k \geqq 1$. The left-side of the equation is 0 on the set $E \cap\left(E+z_{1}\right)$; since $\mu\left(E \cap\left(E+z_{1}\right)\right)>1-1 / 2^{M-1}$, it follows from the induction hypothesis that $c_{i, k}\left[w_{n_{i, k}}\left(z_{1}\right)-1\right]=0$ for each $i \geqq 2$ and all $k$. In particular, taking $i=M$ and $k=1$, we have $-2 c_{M, 1}=0$, since $w_{M_{M, 1}, 1}\left(z_{1}\right)=-1$. Thus $c_{M, 1}=0$. Similarly, we prove that $c_{1,1}=$ $c_{2,1}=\cdots=c_{M-1,1}=0$.

Now suppose that we have shown $c_{i, 1}=c_{i, 2}=\cdots=c_{i, p-1}=0$ for $i=1,2, \cdots, M$, and consider $c_{M, p}$. Let $n_{i, p}(i \neq M)$ be such that 
$n_{M, p}<n_{i, p}$. Choose $z_{p}$ for $n_{i, p}$ so that in the coordinates of $z_{p}$ corresponding to $n_{M, p}$ there are an odd number of 1's, and an even number of 1 's in the coordinates which correspond to each $n_{i, j}(j \geqq p)$. Then, as above, we conclude that $c_{M, p}=0$. Thus $c_{i, k}=0$ for each $i=1,2, \cdots, M$ and every $k \geqq 1$, and hence the given series $S(\boldsymbol{x})$ vanishes identically.

CoRollary. A lacunary Walsh series which converges to 0 (or is constant) almost everywhere vanishes identically.

This latter result is the Walsh analogue of the following important theorem for trigonometric series: a lacunary trigonometric series converging to 0 on a set of positive measure is the identically zero series ([8, vol. I, p. 206]). The statement in the corollary is the best possible in that convergence a.e. cannot be replaced by convergence on a set of measure less than 1 . This follows at once from the fact that in $\left[0,1\left[\left(\right.\right.\right.$ or $\left.2^{\omega}\right)$, the Walsh-Fourier series of the characteristic function of a dyadic interval (basic open set) has only finitely many nonzero coefficients ([7, p. 288]).

The trigonometric result does, however, have the following counterpart in the Walsh system.

THEOREM 2. A lacunary Walsh series which converges to 0 (or is constant) on a set of positive measure has only finitely many nonzero terms.

Proof. The function $\pi: x \rightarrow \mu(E \cap(E+x))$ is continuous ([3, (20. 17)]). Hence if $\boldsymbol{x}$ is 'close' to 0 (that is, if $\boldsymbol{x}$ has sufficiently many 0 's initially), then $\mu(E \cap(E+x))>0$. Let $N$ be the smallest integer for which $E \cap(E+\boldsymbol{x})$ has positive measure whenever $\boldsymbol{x}$ has its first $N$ coordinates equal to 0 .

Let $M$ be the index of 2-lacunarity of the series $S(\boldsymbol{x})$; we prove the theorem by induction on $M$. If $M=1$, let $R$ be the largest power of 2 appearing in the base 2 development of $n_{1}, n_{2}, \cdots, n_{N}$. Define $\boldsymbol{s}_{N+1}$ as follows: let $\boldsymbol{s}_{N+1}$ have 0's in every coordinate up to and including the $R$ th coordinate; an odd number of 1 's in the coordinates which correspond to $n_{N+1}$; an even number of 1 's in the coordinates corresponding to each $n_{j}, j>N+1$; and 0 's in the coordinates not otherwise determined. Then we have

$$
S\left(\boldsymbol{x}+\boldsymbol{s}_{N+1}\right)-S(\boldsymbol{x})=-2 c_{N+1} w_{n_{N+1}}(\boldsymbol{x})
$$

for every $\boldsymbol{x} \in 2^{\omega}$. Since each of $n_{1}, \cdots, n_{N}$ has a power of 2 greater than each power of 2 in its predecessor, it follows that $R \geqq N$, hence $\boldsymbol{s}_{N+1}$ has at least $N$ zeros initially. Thus $E \cap\left(E+\boldsymbol{s}_{N+1}\right)$ is nonempty. As in the proof of the lemma, it can then be shown that $c_{N+1}=0$. 
Proceeding by induction, we conclude that $c_{n}=0$ for every $n>N$.

We retain the notation in the proof of Theorem 1 and suppose the theorem is true for an index $M-1$; that is, if the given series is the sum of $M-1 q$-lacunary series $(q \geqq 2)$ and converges to 0 on a set of positive measure, then $c_{i, j}=0$ for each $i$ and all $j>N$, where $N$ is as in the first paragraph. Consider now the series $S(\boldsymbol{x})=S_{1}(\boldsymbol{x})+\cdots+S_{M}(\boldsymbol{x})$. Let $R$ be the largest power of 2 appearing in the base 2 expansion of $n_{M, 1}, n_{M, 2}, \cdots, n_{M, N}$. Choose $n_{i, J}(i \neq M)$ so that $n_{i, J}>n_{M, N+1}$ and $n_{i, J}$ contains a power of 2 greater than each power of 2 in $n_{M, N+1}$ (this is always possible unless each subsequence $\left\{n_{i, k}\right\}$ is finite, in which case we are done). Define $\boldsymbol{s}_{J}$ for $n_{i, J}$ as follows: let $\boldsymbol{s}_{J}$ have 0's in every coordinate up to and including the $R$ th; let $\boldsymbol{s}_{J}$ have an odd number of 1's in the coordinates corresponding to $n_{M, N+1}$; let $\boldsymbol{s}_{J}$ have an even numer of 1 's in the coordinates which correspond to each $n_{i, j}$, for every $j \geqq J$. Assign 0 to those coordinates not already determined. Then for each $x \in 2^{\omega}$,

$$
\begin{aligned}
S\left(\boldsymbol{x}+\boldsymbol{s}_{J}\right)-S(\boldsymbol{x})= & \sum_{k=1}^{J-1} c_{i, k}\left[w_{n_{i, k}}\left(\boldsymbol{s}_{J}\right)-1\right] w_{n_{i, k}}(\boldsymbol{x}) \\
& +\sum_{\substack{r=1 \\
r \neq i}}^{M}\left[S_{r}\left(\boldsymbol{x}+\boldsymbol{s}_{J}\right)-S_{r}(\boldsymbol{x})\right],
\end{aligned}
$$

since $w_{n_{i, k}}\left(\boldsymbol{s}_{J}\right)=1$ for $k \geqq J$. Because $\boldsymbol{s}_{J}$ has at least $N$ zeros initially, $E \cap\left(E+s_{J}\right)$ has positive measure and so the left-side of this equation vanishes for $\boldsymbol{x}$ in a set of positive measure. Also, since the first sum on the right-side is finite, it is necessarily constant on sets of positive measure. Thus,

$$
\sum_{\substack{r=1 \\ r \neq 1}}^{M}\left[S_{r}\left(\boldsymbol{x}+\boldsymbol{s}_{J}\right)-S_{r}(\boldsymbol{x})\right]=\sum_{\substack{r=1 \\ r \neq i}}^{M} \sum_{m=1}^{\infty} c_{r, m}\left[w_{n_{r, m}}\left(\boldsymbol{s}_{J}\right)-1\right] w_{n_{r, m}}(\boldsymbol{x})
$$

is constant in a set of positive measure and so by the induction hypothesis, $c_{r, m}\left[w_{r_{r, m}}\left(\boldsymbol{s}_{J}\right)-1\right]=0$ for $r=1,2, \cdots, M, r \neq i$, and all $m>N$. In particular, for $r=M$ and $m=N+1$, we have $w_{n_{M H, N+1}}\left(\boldsymbol{s}_{J}\right)=-1$ and therefore $c_{M, N+1}=0$. Proceeding by induction, we show that $c_{M, j}=0$ for every $j>N$. Thus, $S_{M}(\boldsymbol{x})$ is a finite sum.

Write $S_{1}(\boldsymbol{x})+\cdots+S_{M-1}(\boldsymbol{x})=-S_{M}(\boldsymbol{x})$; since $S_{M}(\boldsymbol{x})$ is finite, it assumes constant values on sets of positive measure. The induction hypothesis then implies that each of $S_{1}(\boldsymbol{x}), \cdots, S_{M-1}(\boldsymbol{x})$ is also a finite sum, and so therefore is $S(\boldsymbol{x})$.

The previous proof uses the hypothesis that $E$ has positive measure only to ensure that the set $E \cap(E+\boldsymbol{x})$ is nonempty for sufficiently 'small' $\boldsymbol{x}$. However, even for $E$ of measure zero, this will still be true if $E$ is of the second category and has the property of Baire (see [5, p. 21]). (A set is said to have the property of Baire if it can 
be expressed as the symmetric difference of an open set and a set of the first category.) If a set has the property of Baire, so does its complement ([5, p. 19]); it follows that any residual set (i.e., the complement of a first category set) has the property of Baire. With a slight modification to the proof of Theorem 2, we have the following result.

Theorem 3. Suppose that $E$ is of the second category and has the property of Baire. Then a lacunary Walsh series which converges to 0 (or is constant) on $E$ is necessarily a finite sum. In particular, if $E$ is residual, then the series is identically constant.

Proof. Let $\boldsymbol{s}_{J}$ be defined as in the previous proof. Then $E \cap\left(E+\boldsymbol{s}_{J}\right)$ is of the second category ([5, p. 21]); since the class of all sets having the property of Baire is a $\sigma$-algebra, $E \cap\left(E+s_{J}\right)$ also has the property of Baire. The first sum on the right-side of the equation in the previous proof is constant on dyadic intervals; since $E \cap\left(E+\boldsymbol{s}_{J}\right)$ must meet some one of these intervals in a set of the second category, the series $\sum_{r=1, r \neq i}^{M}\left[S_{r}\left(\boldsymbol{x}+\boldsymbol{s}_{J}\right)-S_{r}(\boldsymbol{x})\right]$ is constant on a second category set having the property of Baire. The proof of Theorem 2 may now be applied, with the obvious modification to the induction hypothesis, to show that the given series is a finite sum.

Finally, if $E$ is residual, then $E$ is dense. Because the series is finite and hence continuous, it can assume only one value.

3. Sets of uniqueness. A set $C$ is called a set of uniqueness for lacunary Walsh series, or a Walsh $U_{L^{-}}$-set, if the only lacunary series converging to 0 on the complement of $C$ is the identically zero series.

Theorem 4. Suppose that $C$ satisfies one of the following:

(i) $C$ is a null set;

(ii) $C$ is of the first category;

(iii) the complement of $C$ is a dense second category set having the property of Baire.

Then $C$ is a Walsh $U_{L}$-set.

Proof. If $C$ satisfies (i), the result follows from the corollary to Theorem 1. Suppose now that (ii) or (iii) holds; in view of Theorem 3 , a lacunary Walsh series converging to 0 on the complement of $C$ is a finite sum and so continuous. Because the complement of $C$ is dense, the series must vanish identically.

None of the conditions in the theorem are necessary. That (i) and 
(ii) are not necessary follows from the fact that a null set may be of either category and that there exist sets of the first category of any given measure. To show that (iii) is not necessary, we take $C$ to be a residual set of measure zero; then $C$ is a $U_{L}$-set in view of the corollary to Theorem 1.

Lastly, we note that a Walsh $U_{L}$-set must have empty interior and so, in particular, cannot be open: for otherwise the Walsh-Fourier series of the characteristic function of a dyadic subinterval, a finite series, would converge to 0 on the complement of the set but not vanish identically.

\section{REFERENCES}

1. N. K. Bary, A Treatise on Trigonometric Series (two volumes), Macmillan, New York, 1964.

2. N. J. Fine, On the Walsh functions, Trans. Amer. Math. Soc., 65 (1949), 372-414.

3. E. Hewitt and K. Ross, Abstract Harmonic Analysis, volume 1, Springer-Verlag, Berlin, 1963.

4. G. W. Morgenthaler, On Walsh-Fourier series, Trans. Amer. Math. Soc., 84 (1957), $472-507$.

5. J. C. Oxtoby, Measure and Category, Springer-Verlag, New York, 1971.

6. R. E. A. C. Paley, A remarkable system of orthogonal functions, Proc. London Math. Soc., 34 (1932), 241-279.

7. A. A. Sneider, On the uniqueness of expansions in Walsh functions, Rec. Math. (Mat. Sbornik) N. S. 24 (66) (1949), 279-300.

8. A. Zygmund, Trigonometric Series, Second Edition, Cambridge University Press, Cambridge, 1968.

Received December 3, 1971 and in revised form June 16, 1972. This research was supported by NRC Grant A8131.

University of British Columbia 



\section{PACIFIC JOURNAL OF MATHEMATICS}

\section{EDITORS}

\author{
H. SAMELson \\ Stanford University \\ Stanford, California 94305 \\ C. R. HоввY \\ University of Washington \\ Seattle, Washington 98105
}

J. DugundJI

Department of Mathematics

University of Southern California

Los Angeles, California 90007

RICHARD ARENS

University of California

Los Angeles, California 90024

\section{ASSOCIATE EDITORS}
E. F. BeCKENBACH
B. H. NEUMANN
F. WOLF
K. YoshidA

\section{SUPPORTING INSTITUTIONS}

UNIVERSITY OF BRITISH COLUMBIA

CALIFORNIA INSTITUTE OF TECHNOLOGY

UNIVERSITY OF CALIFORNIA

MONTANA STATE UNIVERSITY

UNIVERSITY OF NEVADA

NEW MEXICO STATE UNIVERSITY

OREGON STATE UNIVERSITY

UNIVERSITY OF OREGON

OSAKA UNIVERSITY

\author{
UNIVERSITY OF SOUTHERN CALIFORNIA \\ STANFORD UNIVERSITY \\ UNIVERSITY OF TOKYO \\ UNIVERSITY OF UTAH \\ WASHINGTON STATE UNIVERSITY \\ UNIVERSITY OF WASHINGTON

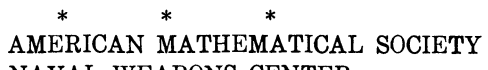 \\ NAVAL WEAPONS CENTER
}

The Supporting Institutions listed above contribute to the cost of publication of this Journal, but they are not owners or publishers and have no responsibility for its content or policies.

Mathematical papers intended for publication in the Pacific Journal of Mathematics should be in typed form or offset-reproduced, (not dittoed), double spaced with large margins. Underline Greek letters in red, German in green, and script in blue. The first paragraph or two must be capable of being used separately as a synopsis of the entire paper. The editorial "we" must not be used in the synopsis, and items of the bibliography should not be cited there unless absolutely necessary, in which case they must be identified by author and Journal, rather than by item number. Manuscripts, in duplicate if possible, may be sent to any one of the four editors. Please classify according to the scheme of Math. Rev. Index to Vol. 39. All other communications to the editors should be addressed to the managing editor, Richard Arens, University of California, Los Angeles, California, 90024.

50 reprints are provided free for each article; additional copies may be obtained at cost in multiples of 50 .

The Pacific Journal of Mathematics is issued monthly as of January 1966. Regular subscription rate: $\$ 48.00$ a year (6 Vols., 12 issues). Special rate: $\$ 24.00$ a year to individual members of supporting institutions.

Subscriptions, orders for back numbers, and changes of address should be sent to Pacific Journal of Mathematics, 103 Highland Boulevard, Berkeley, California, 94708.

PUBLISHED BY PACIFIC JOURNAL OF MATHEMATICS, A NON-PROFIT CORPORATION

Printed at Kokusai Bunken Insatsusha (International Academic Printing Co., Ltd.), 270, 3-chome Totsuka-cho, Shinjuku-ku, Tokyo 160, Japan. 


\section{Pacific Journal of Mathematics}

\section{Vol. 45, No. $2 \quad$ October, 1973}

Kenneth Paul Baclawski and Kenneth Kapp, Induced topologies for quasigroups and loops ............................................. 393

D. G. Bourgin, Fixed point and $\min -\max$ theorems $\ldots \ldots \ldots \ldots \ldots \ldots \ldots$

J. L. Brenner, Zolotarev's theorem on the Legendre symbol ............... 413

Jospeh Atkins Childress, Jr., Restricting isotopies of spheres .............. 415

John Edward Coury, Some results on lacunary Walsh series ................ 419

James B. Derr and N. P. Mukherjee, Generalized Sylow tower groups. II . . . . . . 427

Paul Frazier Duvall, Jr., Peter Fletcher and Robert Allen McCoy, Isotopy Galois

spaces .......................................... 435

Mary Rodriguez Embry, Strictly cyclic operator algebras on a Banach space ... 443

Abi (Abiadbollah) Fattahi, On generalizations of Sylow tower groups ......... 453

Burton I. Fein and Murray M. Schacher, Maximal subfields of tensor products . . 479

Ervin Fried and J. Sichler, Homomorphisms of commutative rings with unit

element .......................................... 485

Kenneth R. Goodearl, Essential products of nonsingular rings ............. 493

George Grätzer, Bjarni Jónsson and H. Lakser, The amalgamation property in

equational classes of modular lattices ...........................

507

$\mathrm{H}$. Groemer, On some mean values associated with a randomly selected simplex

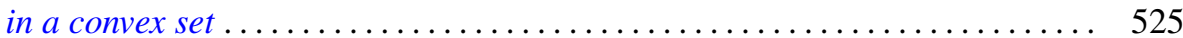

Marcel Herzog, Central 2-Sylow intersections .................... 535

Joel Saul Hillel, On the number of type-k translation-invariant groups ........ 539

Ronald Brian Kirk, A note on the Mackey topology for $\left(C^{b}(X)^{*}, C^{b}(X)\right) \ldots \ldots .543$

J. W. Lea, The peripherality of irreducible elements of lattice.............. 555

John Stewart Locker, Self-adjointness for multi-point differential operators ..... 561

Robert Patrick Martineau, Splitting of group representations ............... 571

Robert Massagli, On a new radical in a topological ring ................. 577

James Murdoch McPherson, Wild arcs in three-space. I. Families of Fox-Artin

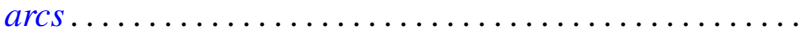

585

James Murdoch McPherson, Wild arcs in three-space. III. An invariant of

oriented local type for exceptional arcs . . . . . . . . . . . . ............ 599

Fred Richman, The constructive theory of countable abelian p-groups ........ 621

Edward Barry Saff and J. L. Walsh, On the convergence of rational functions

which interpolate in the roots of unity ..................

Harold Eugene Schlais, Non-aposyndesis and non-hereditary

decomposability..................................... 643

Mark Lawrence Teply, A class of divisible modules................... 653

Edward Joseph Tully, Jr., H-commutative semigroups in which each

homomorphism is uniquely determined by its kernel ................. 669

Garth William Warner, Jr., Zeta functions on the real general linear group ...... 681

Keith Yale, Cocyles with range $\{ \pm 1\} \ldots \ldots \ldots \ldots \ldots \ldots \ldots \ldots \ldots \ldots \ldots \ldots \ldots . \ldots \ldots$

Chi-Lin Yen, On the rest points of a nonlinear nonexpansive semigroup ........ 699 